\title{
Jóvenes en la ciudad: trayectorias educativas y sociales de los hijos e hijas de familias inmigradas
}

\author{
Pàmies-Rovira, Jordi \\ Departament Pedagogia Sistemàtica i Social \\ EMIGRA CER Migracions - UAB \\ Cerdanyola del Vallès, España \\ jordi.pamies@uab.cat \\ Sánchez-Martí, Angelina \\ Departament Pedagogia Sistemàtica i Social \\ EMIGRA CER Migracions - UAB \\ Cerdanyola del Vallès, España \\ angelina.sanchez@uab.cat \\ Agud-Morell, Ingrid \\ Departament Pedagogia Sistemàtica i Social \\ EMIGRA CER Migracions - UAB \\ Cerdanyola del Vallès, España \\ ingrid.agud@uab.cat
}

\section{Resumen}

En el actual contexto de crisis, ciertos colectivos son más vulnerables y se encuentran expuestos de forma especial a situaciones de desigualdad escolar y social. Este es el caso de los hijos e hijas de familias inmigradas quienes presentan unos elevados índices de paro y fracaso escolar y mayores dificultades para desarrollar procesos de construcción identitaria. La investigación internacional y española ha producido un número significativo de trabajos sobre la desventaja académica de estos jóvenes, y específicamente de ciertos grupos nacionales. Así ocurre también en Cataluña, donde persisten las diferencias con los jóvenes autóctonos, a pesar del mayor tiempo de asentamiento de algunos grupos. Las investigaciones en educación y migraciones han planteado las principales causas de esta situación y las implicaciones que tiene respecto a los procesos de integración y de cohesión social. Sin embargo, las aproximaciones para reconocer las causas del éxito han sido escasas. Esta comunicación presenta los resultados preliminares de un proyecto de investigación que tiene por objetivo conocer la situación de los hijos e hijas de familias inmigradas en un municipio de tamaño medio de Cataluña, donde están presentes 67 nacionalidades y donde la más numerosa es la marroquí. Concretamente y a través de una metodología mixta, el objetivo de la investigación es reconocer las condiciones y posibilidades para el éxito académico y la continuidad educativa entre los jóvenes de origen inmigrante y reconstruir los factores centrales que facilitan el desarrollo de sus trayectorias así como la consolidación de aspiraciones académicas. Para ello, se adapta una perspectiva comparada de tipo «cross-group» (entre grupos) para dilucidar las diferencias entre las nacionalidades más presentes en el estudio.

\section{Abstract}

In the current context of crisis, certain groups are more vulnerable and are exposed especially to situations of school and social in equality. This is the case of the children of immigrant families who show high rates of unemployment and school failure and greater difficulties in developing identity construction processes. International and Spanish research has produced a significant number of works on the academic disadvantage of these young people, and specifically of certain national groups. This is also the case in Catalonia, where differences persist with native youth, despite the longerset tlement times of some groups. Research in education and migration has raised the main causes of this situation and its implications regarding the processes of integration and social cohesion. However, approaches to recognize the causes of success have beens carce. This paper presents the preliminary results of a research project that aims to know the situation of the children of immigrant families in a medium-sized municipality in Catalonia, where 67 nationalities are present and where the largest is Moroccan. Specifically, and through a mixed methodology, the objective of this study is to recognize the conditions and possibilities for academic success and educational continuity among young people of immigrant families and to reconstruct the central factors that facilitate the development of their trajectories as well as the consolidation of their academic aspirations. In doing so, a comparative perspective "cross-group» (among migrant groups) will be adopted to elucidate the differences between the largest nationalities present in the study.

Palabras clave: jóvenes inmigrantes, trayectorias escolares y sociales, éxito y continuidad educativa.

Keywords: young immigrants, school and social trajectories, success and educational continuity. 


\section{INTRODUCCIÓN}

En el actual contexto de crisis, ciertos colectivos son especialmente vulnerables y se encuentran expuestos de forma particular a situaciones de desigualdad escolar y social. Este es el caso de los hijos e hijas de familias inmigradas, un grupo que presenta unos elevados índices de paro y fracaso escolar y a menudo mayores dificultades para poder desarrollar procesos de construcción identitaria. Las investigaciones muestran que en la mayoría de los países existen diferencias significativas entre el rendimiento de estos estudiantes extranjeros y sus iguales nativos (OCDE, 2015a). En el caso de Cataluña, a pesar de la falta de datos oficiales existentes sobre los resultados académicos que obtiene el alumnado extranjero en las diferentes etapas educativas, contamos con investigaciones que les atribuyen, como sucede en otros entornos, unos resultados inferiores, una baja acreditación y unos menores niveles de continuidad postobligatoria que sus iguales nativos. Sin embargo, la constatación de estas tendencias que ponen de relieve la existencia de trayectorias de fracaso, no debería invisibilizar las experiencias de éxito y continuidad educativa que tienen también otros jóvenes extranjeros de ambos sexos en las sociedades que se encuentran (Bertrán, Ponferrada y Pàmies, 2016; Sánchez-Martí, 2016). Ambas situaciones son las que se desean abordar en la propuesta que se presenta y que recoge la experiencia acumulada en investigación básica aplicada en esta línea.

\section{TRAYECTORIAS ESCOLARES DE ÉXITO Y MINORÍAS}

Las investigaciones señalan que, apesar de tener una actitud más positiva hacia la escuela y unas aspiraciones más elevadas los jóvenes extranjeros obtienen peores resultados que sus iguales nativos y que, sus aspiraciones disminuyen con el tiempo mientras que, en especial entre ciertos grupos, no mejoran sus resultados académicos (Kaoy Tienda, 1995; OCDE 2015b, entre otros).

La literatura de investigación sobre migración y educación muestra, asi mismo, como se reinscriben las desigualdades y se distribuyen las oportunidades en la escuela.Y señala, también, que en los diversos sistemas educativos tanto las políticas públicas como la estructura y cultura escolar adquieren formas y significaciones diferenciadas entre los y las jóvenes de minorías y colaboran en la construcción de sus experiencias y trayectorias (Oakes, 1985; Anyon, 1997; Crul y Doomernik, 2003; Dumay y Dupriez, 2009 , ente otros). En particular, señalan que la variabilidad de las experiencias y trayectorias escolares vienen condicionadas, tanto por las políticas públicas - y en especial educativas -, las estructuras de inserción y las prácticas que facilitan o no las transiciones e incorporaciones en los nuevos contextos, como por las percepciones - objetivas u objetivables - que estos jóvenes y sus familias tienen de la estructura de oportunidades y de la realidad social. Estas dimensiones resultan en especial significativas para explicar las experiencias escolares entre los grupos, en especial, aquellos que han tenido una historia de contacto previa con las sociedades de destino (Gibson y Ogbu,1991).

Sin embargo, la constatación de la existencia de unas tendencias de fracaso escolar no puede invisibilizar las trayectorias de éxito y la creciente participación social de una parte considerable de jóvenes inmigrantes e hijos de inmigrantes de ambos sexos. Desde ambas perspectivas y teniendo en cuenta las aportaciones de la literatura de investigación en migraciones y educación, se plantea la investigación que se presenta.

\section{METODOLOGÍA}

Este proyecto, a demanda del Departamento de Ciudadanía de un Ayuntamiento de la provincia de Barcelona, se inscribe en la necesidad de conocer la situación de los jóvenes hijos e hijas de familias inmigradas en el municipio, donde hay 54 nacionalidades y donde la más numerosa es la nacionalidad marroquí. La finalidad del proyecto es conocer las trayectorias y los procesos de integración escolar y social de los hijos e hijas de familias inmigradas, de entre 12 y 24 años, que viven en el municipio.

El diseño metodológico de esta investigación es de carácter mixto. La complejidad de la temática y el carácter holístico de los objetivos perseguidos justifican que la metodología de este estudio responda a un método mixto secuencial (Creswell y Plano Clark, 2007) que incluye diversas técnicas de obtención de información con potencialidades y cualidades complementarias. El estudio se plantea en tres fases diferenciadas y responde a un diseño metodológico mixto de tipo descriptivo-comprensivo con técnicas cuantitativas y cualitativas de recogida y análisis de datos. Las fases del diseño son las siguientes:

- Fase 1. Estudio para encuesta de las trayectorias educativas y sociales de los jóvenes de familia sin migradas. 
En esta fase se ha administrado u ncuestionario dirigido a la población de jóvenes de 12 a 16 años que cursa la educación secundaria obligatoria de todos los centros públicos y privados del municipio. El instrumento de medida ha sido adaptado a partir de un cuestionario de uno de los antecedentes director de este estudio (RESL.eu). Originariamente este instrumento estaba dirigido a recoger las experiencias y monitorizar las trayectorias de los jóvenes que llegan al final de la etapa de educación obligatoria para identificar posibles factores de riesgo que pueden derivar en procesos de abandono escolar prematuro. El instrumento fue administrado en siete estados miembro de la UE. Muchos de los indicadores recogidos mediante este cuestionario responden a los objetivos específicos aquí detallados, por lo que se estimó conveniente su adaptación en este estudio. Asi mismo,se concibe como una oportunidad para comparar ciertos indicadores con datos internacionales.

La aplicación de los cuestionarios a todos los centros educativos del municipio estaba planificado para los meses de marzo-mayo de 2018. Finalmente, la aplicación del mismo se ha iniciado en el mes de mayo, por lo que todavía no se cuenta con datos suficientes para hablar de resultados preliminares.

- Fase2.Análisis en profundidad de las trayectorias educativas y sociales de los jóvenes de familia sin migradas.

Fruto del análisis anterior, está prevista una segunda fase cualitativa en la que se prevé la realización de historias de vida a un número adecuado de los jóvenes y grupos de discusión de jóvenes y profesionales de la educación, representantes de asociaciones, y técnicos del ámbito municipal. La realización de historias de vida y del método biográfico interpretativo (Breckner, 2000; Fischer-Rosenthal, 2000, entre otros) debe permitir tanto contextualizar su experiencia narrativa en el ámbito de la escuela como en el de las transiciones y las relaciones inter e intraculturales. En todos estos ámbitos la incidencia de las políticas, culturas y prácticas (educativas, laborales, sociales, etc.) son un elemento clave en tanto que establecen las condiciones para y de la integración. Y a su vez articulan como referentes los dispositivos a implementar. Valorar el significado objetivo y objetivable que estas políticas, culturas y prácticas han tenido en sus procesos de integración social y en sus trayectorias vitales se convierte en un elemento clave que da sentido a los resultados que han obtenido y nos habla de las posibilidades y también de los límites existentes en la sociedad catalana.

Para completar esta aproximación se realizarán diferentes grupos de discusión con agentes implicados en su trayectoria tanto desde la vertiente intragrupo como exogrupo. Concretamente, está previsto realizar grupos de discusión con miembros de asociaciones, tanto culturales como religiosas, profesionales vinculados a los dispositivos para la acogida, integración y cohesión social, así como con docentes y profesionales vinculados a la escuela y otros dispositivos para la acogida o apoyo educativo del municipio.

- Fase 3. Elaboración de propuestas de mejora y de intervención educativa y social.

En esta fase se llevará a cabo la integración de los resultados obtenidos en las dos fases anteriores, los cuales serán recogidos en el informe final del estudio y, a partir de los cuales, surgen las propuestas y orientaciones de intervención educativa y social.

\section{RESULTADOS}

Los resultados de este estudio permitirán:

- describir las trayectorias escolares y sociales de los hijos e hijas de familias inmigradas en el municipio donde se ha llevado a cabo el estudio.

- conocer los factores que inciden de forma positiva y aquellos que han actuado y actúan como factores limitantes en los procesos de integración escolar y social de estos chicos y chicas.

- identificar las características -escolares y sociales- que comparten los chicos/as hijos de familias inmigradas con trayectorias de éxito y establecer el vínculo con el papel de las instituciones -y los agentes- de educación formal y no formal del municipio.

- identificar las condiciones de la institución escolar -estructura y cultura- en las que se desarrollan y se han desarrollado las trayectorias de los hijos e hijas de familias inmigradas.

- reconocer el grado de identificación y los puntos que pueden devenir de conflicto en el caso de los hijos e hijas de familias inmigradas.

- hacer propuestas de mejora dirigidas al desarrollo de políticas públicas y educativas a partir de los factores de éxito identificados en las trayectorias académicas y sociales de estos chicos y chicas. 


\section{DISCUSIÓN Y CONCLUSIONES}

En Cataluña, aunque existen escasos datos al respecto, sabemos que hay chicos y chicas de origen extranjero que obtienen peores resultados que sus iguales autóctonos al fianlizar la ESO y tienen una menor continuidad académica (Serra y Palaudàriuas, 2007), llegándose a naturalizar la situación como algo propio de estos grupos (Abajo y Carrasco, 2004; Pàmies, 2011). Estos chicos y chicas de origen inmigrante a lo largo de su escolarización con mayor frecuencia son encuadrados en grupos escolares de menor prestigio, y orientados, como ocurre en otros países europeos, hacia itinerarios de formación profesional y ocupacional (Carrasco, Ruiz-Haro y Bereményi, 2018). Pero como han mostrado investigaciones anteriores (Pàmies y Bertran, 2016; Sánchez-Marti, 2016) cuando consiguen trazar trayectorias de éxito y continuidad educativa, coinciden una serie de dimensiones: el apoyo de la familia, el capital social del grupo de iguales, una experiencia escolar positiva y la figura de un profesor que acompaña y ayuda a superar las situaciones de crisis, y el apoyo ofrecido por las asociaciones inter o intraétnicas.

Resulta conveniente pues que en el estudio de la situación educativa de los hijos e hijas de inmigrantes, además de ampliar el corpus de conocimiento en la línea del fracaso, que ha descrito el fenómeno y ha buscado las causas, introduzcamos la perspectiva centrada en las condiciones para el éxito, desde una perspectiva sociopedagógica. Con este objetivo se desarrolla esta investigación que viene a completar los datos obtenidos en proyectos de investigación básica y aplicada anteriores en los ámbitos escolares, familiares y comunitarios centrados en los hijos e hijas de familias inmigrantes en Cataluña.

\section{REFERENCIAS BIBLIOGRÁFICAS}

Abajo, J. E. y Carrasco, S. (2004). Experiencias y trayectorias de éxito escolar de gitanas y gitanos en España. Madrid: CIDE/Instituto de la Mujer.

Anyon, J. (1997). Ghetto schooling: A political economy of urban educational reform. New York: Teachers College Press.

Bertran, M., Ponferrada, M. y Pàmies, J. (2016). Gender, family negotiations and academic success of young Moroccan women in Spain. Race Ethnicity \& Education, 19(1), 161-181.

Breckner, R. (2000). The Meaning of the 'Iron Curtain' in East-West Migration Biographies, in: R. Breckner, D. Kalekin-Fishman und I. Miethe (Hg.) Biographies and the Division of Europe. Experience, Action and Change on the 'Eastern Side' (pp.367-387).Opladen:Leske+Budrich.

Carrasco, S., Ruiz-Haro, I. y Bereményi, B. Á. (2018). No bridges to reengament? Exploring compensatory measures for early school leavers in Catalonia (Spain) from a qualitative approach. En Ch.Timmermann, L.Van Praag \& N. Clycq, eds., Reducing Early School Leaving in the EU. A Quantitative and Qualitative Study. London: Routledge (enprensa).

Creswell, J. y Plano Clark, V. (2007). Designing and Conducting Mixed Methods Research. Thousand Oaks, CA: SageCrul y Doomernik.

Dumay, X. y Dupriez, V. (ed.) (2009). L'efficacité dans l'enseignement, promesses et zones d'ombre. Bruselas: De Boeck.

Fischer-Rosenthal, W. (2000). Address lost: How to Fix Lives. Biographical Structuring in the EuropeanModernAge.En R. Breckneret.al.(Hg.) Biographies and the Division of Europe (pp. 55-75). Opladen: Leske +Budrich.

Gibson, M. y Ogbu, J. (eds.) (1996). Minority status and schooling: A comparative study of immigrant and Voluntary Minorities. Nueva York: Garland Publishing.

Kao, G. y Tienda, M. (1995). Optimism and achievement: the educational performance of immigrants youth, Sociological Quarterly, 76 (1), 1-19.

Oakes, J (1985 [2005]). Keeping Track: How Schools Structure Inequality. Yale University Press OCDE (2015a). Helping immigrant students to succeed at school - and beyond. Paris.

OCDE (2015b). Immigrant Students at School. Easing the Journey towards Integration. Paris.

Pàmies, J. (2011). Moroccan immigrants at a secondary school in Catalonia. En W. Berg, J, Spinthouralis \& J. Lalor (dir). Cultural diversity in the Classroom. Springer VS: Verlag f̈̈r Sozialwissenschaften.

Sánchez-Martí, A (2016). Trajectòries d'èxit educatiu dels joves de famílies immigrades a secundària postobligatòria: centres educatius i agents institucionals. [Tesis doctoral]. Barcelona: Universitat de Barcelona. Departamento de Métodos de Investigación y Diagnóstico en Educación.

Serra, C. y Palaudàrias, J. M. (2007). L'alumnat de nacionalitat estrangera en els estudis postobligtoris. En M.J. Larios y M. Nadal (dir.), L'estat de la immigració a Catalunya. Anuari 2006 (pp. 301-334). Barcelona: Mediterrània. 